\title{
Predicting the Effective Temperature of a Glass
}

\author{
Nicoletta Gnan, ${ }^{*}$ Claudio Maggi, Thomas B. Schrøder, and Jeppe C. Dyre \\ DNRF Center "Glass and Time," IMFUFA, Department of Sciences, Roskilde University, P.O. Box 260, DK-4000 Roskilde, Denmark
} (Received 23 December 2009; published 24 March 2010)

\begin{abstract}
We explain the findings by Di Leonardo et al. [Phys. Rev. Lett. 84, 6054 (2000)] that the effective temperature of a Lennard-Jones glass depends only on the final density in the volume and/or temperature jump that produces the glass. This is not only a property of the Lennard-Jones liquid, but a feature of all strongly correlating liquids. For such liquids data from a single quench simulation provide enough information to predict the effective temperature of any glass produced by jumping from an equilibrium state. This prediction is validated by simulations of the Kob-Andersen binary Lennard-Jones liquid and shown not to apply for the nonstrongly correlating monatomic Lennard-Jones Gaussian liquid.
\end{abstract}

DOI: 10.1103/PhysRevLett.104.125902

PACS numbers: $65.20 .-\mathrm{w}$

Condensed matter is frequently found in out-ofequilibrium states. For example, for systems like supercooled liquids, dense colloids, spin systems, etc., the (offequilibrium) glass state occurs naturally after cooling or compression from a state of thermal equilibrium. An effective temperature describes the nonequilibrium properties of a glass, and the possibility of connecting the effective temperature with the observed violation of the fluctuation-dissipation theorem (FDT) [1] has opened new ways of inquiry [2-6]. In 2000, Di Leonardo et al. [5] studied the off-equilibrium dynamics of the singlecomponent Lennard-Jones (LJ) liquid (with a small many-body term added to the potential to prevent crystallization). This system was subjected to sudden temperature decreases at constant density (quenches) as well as to sudden density increases at constant temperature (crunches). From the violation of the FDT, the effective temperature was determined. Surprisingly, it was observed that the effective temperature $T_{\text {eff }}$ is independent of the particular path in the temperature-density plane crossing the glass transition line: $T_{\text {eff }}$ depends only on the final density. In this Letter we demonstrate that the findings of Di Leonardo et al. hold generally for strongly correlating liquids (defined below). We further argue and demonstrate that - for this class of liquids - from a single quench simulation one can predict the effective temperature for any offequilibrium jump.

Reference [7] documented the existence of a large class of liquids characterized by strong correlations between virial $\left(W \equiv p V-N k_{B} T\right)$ and potential energy $(U)$ thermal equilibrium fluctuations at fixed volume, $\Delta W(t) \cong$ $\gamma \Delta U(t)$. Strongly correlating liquids have a hidden (approximate) scale invariance, which implies that they inherit many-but not all—of the scaling properties of liquids interacting via inverse power-law potentials. Strongly correlating liquids include van der Waals-type liquids but not, e.g., hydrogen-bonding liquids. Strongly correlating liquids have curves in their phase diagrams- "isomorphs"-along which several static and dynamic prop- erties are invariant [8]. These invariants derive from the fact that two microscopic configurations of two isomorphic state points, which scale into one another, to a good approximation have identical canonical probabilities. If the density is denoted by $\rho$, an isomorph is given by $\rho^{\gamma} / T=$ const. The exponent $\gamma$-which may be slightly state-point dependent — can be calculated from equilibrium fluctuations at one state point or from a single quench simulation utilizing the relation between the relaxing averages, $\langle W(t)\rangle \cong \gamma\langle U(t)\rangle+W_{0}$.

Because the canonical probabilities of scaled configurations belonging to the same isomorph are identical, a jump between two isomorphic state points takes the system instantaneously to equilibrium [property (i)][8]. Moreover, jumps from isomorphic state points to the same final state point show identical aging behavior [property (ii)] [8]. In view of these properties the results of Di Leonardo et al. [5] may be understood as follows. A crunch from density $\rho_{1}$ to density $\rho_{2}$ can be ideally decomposed into two parts (Fig. 1): First, the system jumps instantaneously from its initial state to the corresponding isomorphic state at the final density (i.e., the state which has the same $\rho^{\gamma} / T$ as the initial state); see Fig. 1. This is an equilibrium state [8]. Thereafter the system at constant density begins to approach the equilibrium state defined by the temperature. If the crunch is made to a state with very high density, the thermalization takes an extremely long time and the effective temperature may be determined from the FDT violation as detailed below. In this way any crunch corresponds to a quench to the final density with the same relaxation pattern. In particular, these two transformations should have identical FDT violation factors and identical effective temperatures.

These arguments should apply to any strongly correlating liquid, not just the single-component LJ system. To confirm this we simulated the Kob-Andersen binary Lennard-Jones (KABLJ) liquid [9,10]. Following Di Leonardo et al. [5] we subjected the KABLJ liquid to a number of instantaneous quenches and crunches and 


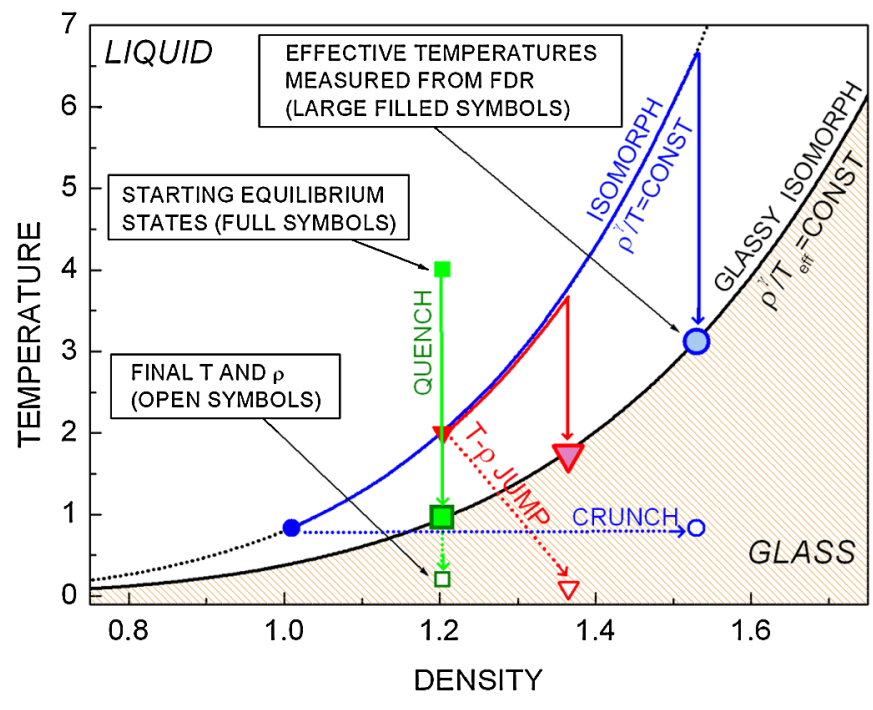

FIG. 1 (color online). Patterns followed by the KABLJ liquid in different off-equilibrium density and/or temperature jumps. Consider, for example, the case of a crunch (horizontal dotted line), where the system is densified at constant temperature. This transformation is equivalent to a quench (right-most vertical line) from an isomorphic state point having the final density of the crunch. Thus the $T_{\text {eff }}$ (large filled circle) is identical for these transformations. In all processes represented here the liquid undergoes a glass transition characterized by an effective temperature that can be measured from the fluctuation-dissipation relation (FDR) Eq. (1). $T_{\text {eff }}$ versus (final) density constitutes an isomorph, as discussed later in the text.

calculated the effective temperatures from the fluctuationdissipation relation (FDR). Recall that for off-equilibrium systems the FDR in $k_{B}=1$ units is [11-15]

$$
T \partial_{t^{\prime}} \chi\left(t, t^{\prime}\right)=-X\left(t, t^{\prime}\right) \partial_{t^{\prime}} C\left(t, t^{\prime}\right) .
$$

Here $C=\left\langle A(t) B\left(t^{\prime}\right)\right\rangle$ is the correlation function of the variables $A$ and $B$ in the unperturbed situation, the perturbing contribution to the Hamiltonian is $\delta H=-\epsilon B$, $\chi\left(t, t^{\prime}\right)=\langle A(t)\rangle /\left.\epsilon\right|_{\varepsilon \rightarrow 0}$ is the response of $A$ to the perturbation applied at time $t^{\prime}<t$, and $X$ is the FDT violation factor. This is unity at short times $\left(t-t^{\prime}\right) / t^{\prime} \ll 1$, while $X<1$ in the long-time limit $\left(t-t^{\prime}\right) / t^{\prime} \gg 1$. We chose as dynamic variables $A_{\mathbf{k}}(t)=N^{-1} \sum_{j} \eta_{j} \cos \left[\mathbf{k} \cdot \mathbf{r}_{j}(t)\right]$ and $B_{\mathbf{k}}(t)=2 N A_{\mathbf{k}}(t)$, where the sum is extended to all $N$ particles of the system and $\eta_{j}= \pm 1$ is a random variable with zero mean. With this choice the correlation function $C\left(t, t^{\prime}\right)$ is the self-intermediate scattering function.

For quenches to low enough temperatures, at long times an effective temperature of the slow degrees of freedom is associated with the FDT violation factor: $T_{\text {eff }}=T / X[12-$ 16]. The effective temperature reflects the slow structural rearrangements in the sense that the aging system behaves as if it were thermalized at $T_{\text {eff }}$ [16]. We obtained $X$ by calculating the correlation function and the response function in the nonequilibrium regime by means of $X=X(t)=$ $-T \partial \chi\left(t, t^{\prime}\right) /\left.\partial C\left(t, t^{\prime}\right)\right|_{t}$, which applies at long times (note that the correct $X$ is found by taking this derivative at fixed $t$, not at fixed $\left.t^{\prime}[17]\right)$.

Recently Berthier introduced a new method for calculating the response without applying an external field for an off-equilibrium Monte Carlo simulation of the KABLJ [17]. Using his procedure Fig. 2 shows the FD plots for the KABLJ liquid during a number of temperature-density jumps. In Fig. 2 we test the construction of equivalent crunches and quenches argued above: a crunch and a quench from initially isomorphic states (i.e., with the same $\rho^{\gamma} / T$ ) to the same final $T$ and $\rho$ (red circles and red squares). Clearly the crunch overlaps well with the quench; in fact, they follow the same aging pattern. The exponent $\gamma$ was estimated by a linear fit of the parameter plot $\langle W(t)\rangle$ vs $\langle U(t)\rangle$ when the system is relaxing after a temperature jump from $T=2.55$ to $T=0.3192$ at fixed $\rho=1.264$. The resulting value is $\gamma=5.01$ (for details, refer to Ref. [10]). Figure 3 shows the linear relation that connects $\langle W(t)\rangle$ and $\langle U(t)\rangle$ during two "isomorphic" quenches.

Identical responses and correlations do not only appear when a strongly correlating liquid is taken from two isomorphic states to the same state point. Supplementing properties (i) and (ii), strongly correlating liquids have a

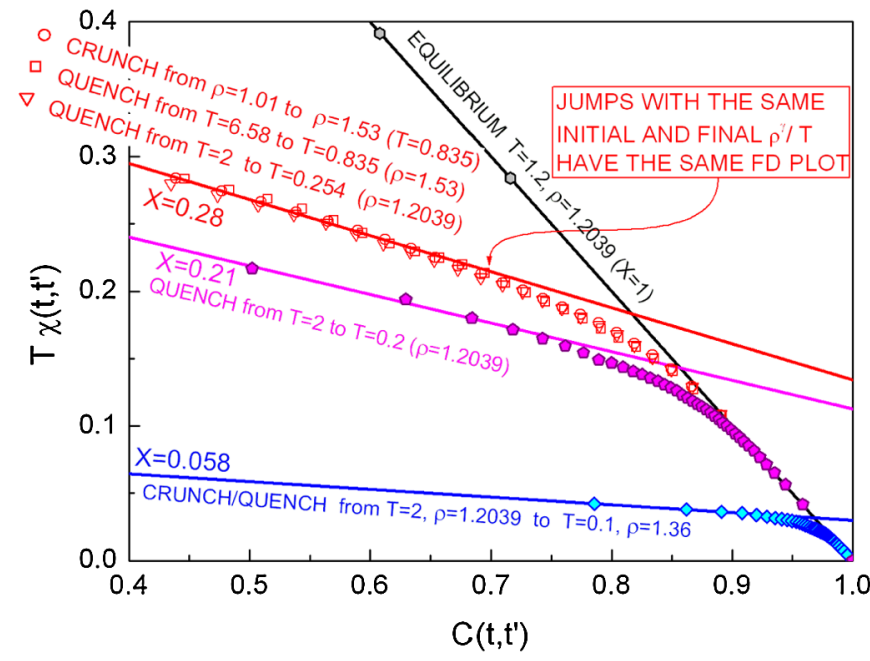

FIG. 2 (color online). Response versus correlation function for several density or temperature jumps for the KABLJ liquid. All FD plots have fixed $t=10^{4}$ (Monte Carlo steps) and $t^{\prime}$ varying from $10^{3}$ to $10^{4}$. All functions plotted here have the same reduced k-vector (referring to the final density) and the same reduced microscopic time. In the crunch $(\bigcirc)$ we set $|\mathbf{k}|=7.81$ corresponding to the reduced k-vector $|\tilde{\mathbf{k}}|=6.78$ (see Ref. [10] for details). The crunch $(\bigcirc)$ overlaps very well with the quench $(\square)$ that takes the system from an initial state isomorphic to the one of the crunch to the same final state. Note also the good superposition of the additional quench $(\nabla)$ that takes the system from a state isomorphic to the initial state of $(\bigcirc)$ to a state isomorphic to the final one of $(\bigcirc)$. The full lines have the slopes predicted from the density-scaling relation Eq. (2) for $T_{\text {eff }}$. 


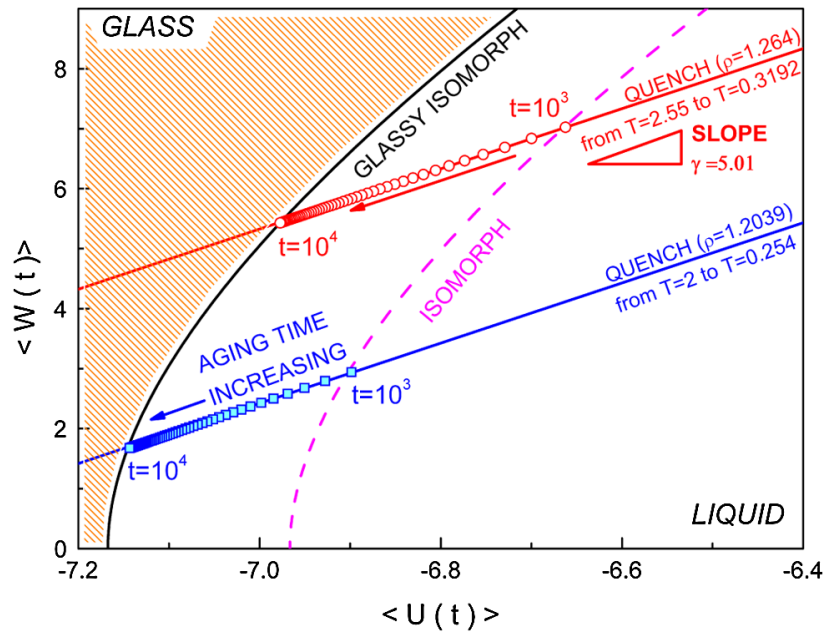

FIG. 3 (color online). Average virial versus potential energy per particle during aging in two quenches for the KABLJ liquid. These quenches were performed between states with same initial and final $\rho^{\gamma} / T$. At each time the off-equilibrium states are connected by an isomorph (e.g., the dashed line). In these two jumps the FDT violation factor $X$ should be identical. The slope of the $\langle W(t)\rangle$ vs $\langle U(t)\rangle$ plot is $\gamma$. The analytical equation used for drawing the isomorphs in the $W-U$ plot here is reported in Ref. [20].

third interesting aging property (iii): For two jumps $\left(T_{1}, \rho_{1}\right) \rightarrow\left(T_{2}, \rho_{2}\right)$ and $\left(T_{3}, \rho_{3}\right) \rightarrow\left(T_{4}, \rho_{4}\right)$ between mutually isomorphic initial and final states (i.e., $\rho_{1}^{\gamma} / T_{1}=$ $\rho_{3}^{\gamma} / T_{3}$ and $\rho_{2}^{\gamma} / T_{2}=\rho_{4}^{\gamma} / T_{4}$ ), the systems follow the same path in configuration space in reduced units [10] because the dynamical equations governing the evolution of the particle trajectories are identical in reduced units. Accordingly, the responses and correlations of two such jumps must be identical in reduced units. In Fig. 2 we show the reduced unit $C$ and $\chi$ of a quench between initial and final states that are isomorphic, respectively, to the initial and final states of the crunch described above (red triangles). The overlap between the functions is good. Figure 3 shows the variables $\langle W(t)\rangle$ vs $\langle U(t)\rangle$ in two such isomorphic jumps; they are connected by an isomorph at each time during the relaxation.

A further consequence of property (iii) is the following. Because the reduced-unit evolution is the same for the system in the two jumps, their FDR violation factors must also be identical, $X_{2}=X_{4}$. Combining this equation with $\rho_{2}^{\gamma} / T_{2}=\rho_{4}^{\gamma} / T_{4}$ and expressing $X$ via the effective temperature, we find $\rho_{2}^{\gamma} / T_{\text {eff, } 2}=\rho_{4}^{\gamma} / T_{\text {eff, } 4}$, i.e.,

$$
\rho^{\gamma} / T_{\text {eff }}=\text { const. }
$$

This equation identifies the glass transition curve in the $(T, \rho)$ plane defined in terms of the FDR effective temperature with an isomorph. This is consistent with the findings of Ref. [5] and the standard way of defining the glass transition, because the standard glass line in the $(T, \rho)$ plane is located where the equilibrium relaxation time reaches a certain (high) value of order the inverse cooling rate. For strongly correlating liquids an isomorph is also an "isochronal" curve along which the (reduced) relaxation time is constant [8]. Figure 2 shows the slopes predicted by Eq. (2) (lines); clearly the prediction is fulfilled.

It is well known (see, for example, Refs. $[4,17]$ ) that the effective temperature is independent of the initial and final temperature if the initial temperature is high (the system is in a warm liquid state) and if the quenching temperature is low enough (i.e., in the regime where $X=T / T_{\text {eff }}$ with constant $T_{\text {eff }}$ ). Consequently, Eq. (2) predicts the effective temperatures for all possible jumps ending at density $\rho$. The exponent $\gamma$ and the constant may both be calculated from the results of one single aging simulation. In Fig. 4 we compare $T_{\text {eff }}$ identified from several crunches and quenches (not only involving isomorphic initial and final state points) with the prediction of Eq. (2). The agreement is very good.

The above discussed simple aging properties are only expected to apply for liquids with isomorphs, i.e., strongly correlating liquids. To validate this we simulated the nonequilibrium dynamics of the monatomic Lennard-Jones Gaussian (MLJG) model [18]. The pair potential of the MLJG has an additional Gaussian attractive well compared to the LJ liquid (see the inset of Fig. 5); details about the model's potential and its glassy behavior can be found in Ref. [18]. The MLJG liquid has $W U$ fluctuations which correlate less than $2 \%$ at the state points studied here. As is clear from Fig. 5, two jumps to the same final density lead to quite different effective temperatures. Thus, this system provides a counterexample to the observation by

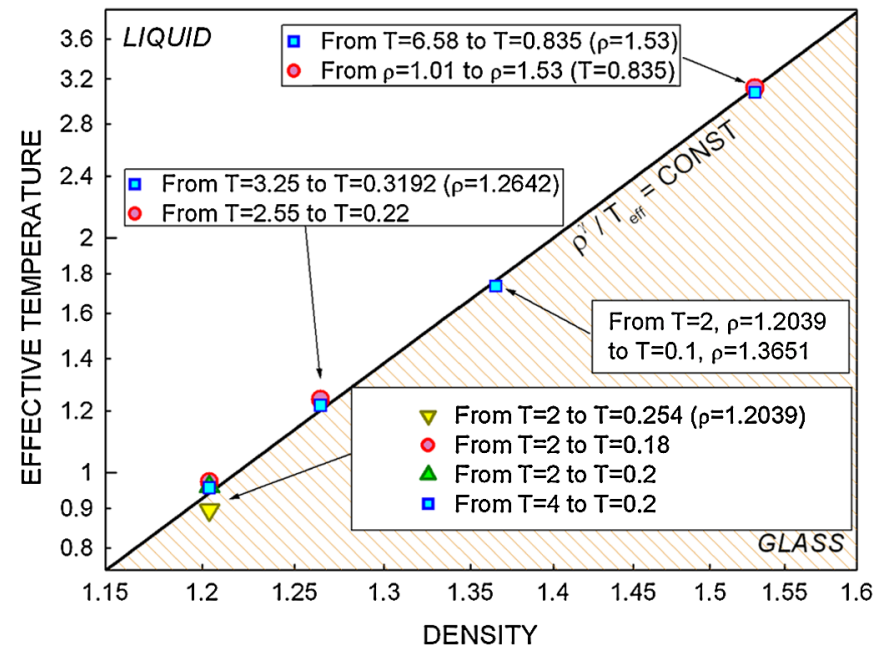

FIG. 4 (color online). Effective temperature as a function of density in several crunch and/or quenches (in double log scale) for the KABLJ liquid. The effective temperature is computed from the violation factor: $T / T_{\text {eff }}=X=-T \partial \chi\left(t, t^{\prime}\right) /\left.\partial C\left(t, t^{\prime}\right)\right|_{t}$. The scaling exponent $\gamma$ is computed from potential energy-virial relaxation (see Fig. 3) as described in the text. The full line is the prediction of the density scaling equation (2) for $T_{\text {eff }}$. 


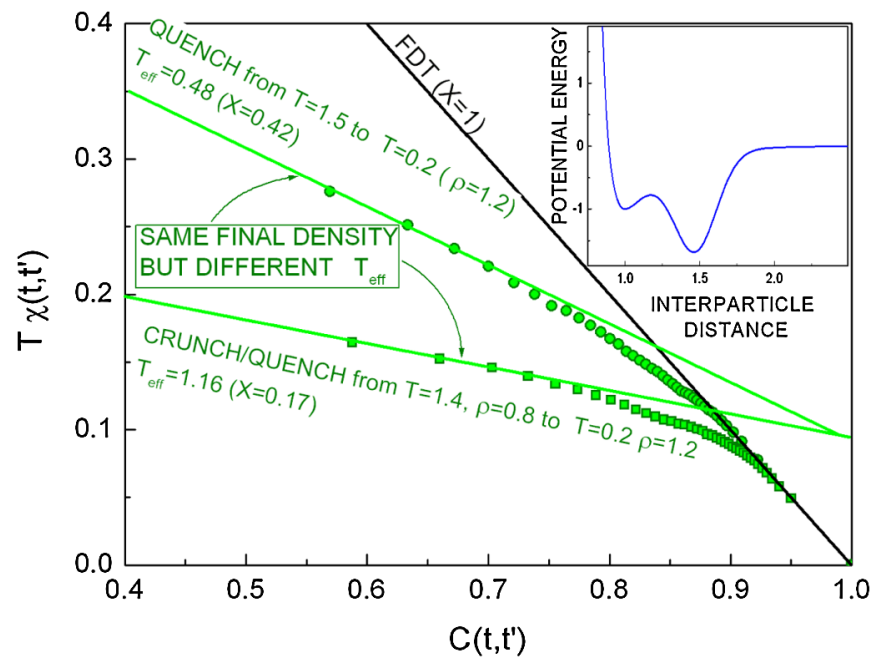

FIG. 5 (color online). FD plot for two jumps of the MLJG liquid ending at same final density and temperature. The potential defining this model is shown in the inset. In this case the two effective temperatures are very different.

Di Leonardo et al. [5] that the effective temperature depends only on the final density.

We also investigated the relation between the inherent state energies in aging and at equilibrium for the KABLJ and the MLJG liquids (see Ref. [10] for more details). Only for the strongly correlating liquid KABLJ can one interpret $T_{\text {eff }}$ as an indicator of which part of the energy landscape is visited during aging, confirming a suggestion by Sciortino and Tartaglia in [19].

In conclusion, the existence of isomorphs for strongly correlating liquids explains the previously reported result [5] for the LJ liquid that the effective temperature depends only on the final density of any jump (when temperature and density are the externally controlled variables). We presented simulations of the aging dynamics of another strongly correlating liquid, the KABLJ liquid, as well as simulations of aging of a liquid without strong virial or potential energy correlations (the MLJG liquid). For strongly correlating liquids it is always possible to produce equivalent density or temperature transformations connected by the density-scaling relation. Moreover, for this class of liquids the effective temperature satisfies the density-scaling equation (2). Since the exponent $\gamma$ and the constant of Eq. (2) may both be identified from a single quench simulation, the implication is that for a strongly correlating liquid the effective temperature of an arbitrary glass may be calculated from the results of a single jump simulation.

The center for viscous liquid dynamics Glass and Time is sponsored by the Danish National Research Foundation (DNRF).

*ngnan@ruc.dk

[1] J.-P. Hansen and I. R. Mc-Donald, Theory of Simple Liquids (Academic, New York, 2006).

[2] G. Parisi, Phys. Rev. Lett. 79, 3660 (1997).

[3] M. Sellitto, Eur. Phys. J. B 4, 135 (1998).

[4] W. Kob and J. L. Barrat, Europhys. Lett. 46, 637 (1999).

[5] R. Di Leonardo, L. Angelani, G. Parisi, and G. Ruocco, Phys. Rev. Lett. 84, 6054 (2000).

[6] R. L. Jack, M. F. Hagan, and D. Chandler, Phys. Rev. E 76, 021119 (2007).

[7] U. R. Pedersen et al., Phys. Rev. Lett. 100, 015701 (2008); Phys. Rev. E 77, 011201 (2008); N. P. Bailey et al., J. Chem. Phys. 129, 184507 (2008); 129, 184508 (2008); T. B. Schrøder et al., Phys. Rev. E 80, 041502 (2009).

[8] N. Gnan, T. B. Schrøder, U. R. Pedersen, N. P. Bailey, and J. C. Dyre, J. Chem. Phys. 131, 234504 (2009).

[9] W. Kob and H. C. Andersen, Phys. Rev. Lett. 73, 1376 (1994); L. Berthier and W. Kob, J. Phys. Condens. Matter 19, 205130 (2007).

[10] See supplementary material at http://link.aps.org/ supplemental/10.1103/PhysRevLett.104.125902 for simulation details, definitions of the reduced units, and the study of the off-equilibrium landscape in the two systems studied.

[11] U. M. Marconi, A. Puglisi, L. Rondoni, and A. Vulpiani, Phys. Rep. 461, 111 (2008).

[12] J. Kurchan, Nature (London) 433, 222 (2005).

[13] A. Crisanti and R. Ritort, J. Phys. A 36, R181 (2003).

[14] L. F. Cugliandolo, Slow Relaxations and Nonequilibrium Dynamics in Condensed Matter, Dynamics of Glassy Systems Course 7 (Springer, Berlin, 2004).

[15] C. Chamon and L. F. Cugliandolo, J. Stat. Mech. (2007) P07022.

[16] L. Leuzzi, J. Non-Cryst. Solids 355, 686 (2009).

[17] L. Berthier, Phys. Rev. Lett. 98, 220601 (2007).

[18] V. Van Hoang and T. Odagaki, Physica (Amsterdam) 403B, 1803 (2008).

[19] F. Sciortino and P. Tartaglia, Phys. Rev. Lett. 86, 107 (2001).

[20] N. Gnan et al., arXiv:0905.3497v1. 